\title{
Feature tracking derived longitudinal and circumferential myocardial strain abnormalities in clinical myocarditis
}

\author{
Justin D Weigand ${ }^{1 *}$, James Nielsen ${ }^{2}$, Partho Sengupta ${ }^{3}$, Javier Sanz ${ }^{3}$, Shubhika Srivastava ${ }^{1}$, Santosh Uppu ${ }^{1}$ \\ From 18th Annual SCMR Scientific Sessions \\ Nice, France. 4-7 February 2015
}

\section{Background}

Cardiac magnetic resonance imaging (CMR) is frequently used to assess myocardial involvement to confirm diagnosis of myocarditis. CMR techniques employ T2- weighted, early and late gadolinium enhancement (LGE), with two positive sequences widely considered diagnostic for myocardial inflammation. However, these techniques are qualitative and subjective, thus prone to interpretation error. Feature tracking (FT) allows quantitative segmental myocardial strain analysis using traditional cine CMR images. Our hypothesis was that left ventricular (LV) segmental strain would be a more sensitive indicator for myocardial involvement in patients with clinical myocarditis. Regional strain was compared to presence of LGE as a measure of myocardial involvement.

\section{Methods}

Patients with clinical diagnosis of myocarditis and structurally normal hearts who underwent CMR at our institution from 2004-2014 were studied retrospectively. Consecutive healthy young adults with normal cardiac anatomy, function and absent LGE were selected as controls. FT was performed on CMR cine images; longitudinal strain analysis was performed in four chamber (4C) and two chamber (2C) images, circumferential strain was calculated at basal, midventricular (mid), and apical levels. Global and segmental strain data was compared to troponin leak and presence of LGE. Sensitivity, specificity, and criterion values of strain were determined by comparison to controls. Descriptive statistics, t-tests, logistic regression analysis, and receiver operator characteristic curves were utilized.

\section{Results}

Twenty-nine myocarditis patients and nineteen controls were analyzed by FT. All myocarditis patients demonstrated abnormal myocardial strain in at least one longitudinal or circumferential distribution including patients in whom LGE was negative. Twenty of the 29 myocarditis patients with normal ejection fraction were included in the final analysis. Myocarditis patients had significantly diminished longitudinal $4 \mathrm{C}$, longitudinal $2 \mathrm{C}$, global circumferential, circumferential basal, mid, and apical strain when compared with normal controls (Table 1). Multivariate logistic regression analysis identified longitudinal $4 \mathrm{C}$ strain as the most predictive for troponin leak and LGE $(\mathrm{p}=<0.01$ respectively). Longitudinal $4 \mathrm{C}$ and global circumferential strain demonstrates good sensitivity and specificity for troponin leak and LGE. Circumferential basal and mid ventricular strain demonstrates excellent sensitivity for troponin leak and LGE. Circumferential apical strain has good specificity for troponin leak (Table 2).

\section{Conclusions}

FT derived longitudinal and circumferential strain is more sensitive and specific in identifying myocardial involvement. FT can be a useful adjunct to traditional CMR techniques as an objective measure of myocardial involvement especially in patients with myocarditis and normal LV function. Feature tracking may prove advantageous in cases of clinical myocarditis where traditional CMR techniques are inconclusive.

\section{Funding}

N/A. 
Table 1 Summary statistics

\begin{tabular}{cccc}
\hline & Control & Myocarditis with Normal Function & p-value \\
\hline$N$ & 19 & 20 & $18(17-18.6)$ \\
\hline Median Age (years, IQR) & $19(18-20)$ & $18: 2$ & 0.36 \\
\hline Male:Female & $13: 6$ & 2 & $<0.01$ \\
\hline Mean BSA (m <2>) & 1.9 & 19 & 0.32 \\
\hline Elevated Troponin & 0 & 18 & $<0.01$ \\
\hline Mean LV EF (\%) & 61 & $-14.6 \pm 3.5$ & $<0.01$ \\
\hline$(+)$ LGE & 0 & $-15.2 \pm 5.1$ & 0.03 \\
\hline Longitudinal 4C Strain & $-18.4 \pm 3.9$ & $-17.9 \pm 3.5$ & $<0.01$ \\
\hline Longitudinal 2C Strain & $-18.7 \pm 4.9$ & $-18.2 \pm 4.6$ & $<0.01$ \\
\hline Global Circumferential Strain & $-22.7 \pm 3.2$ & $-17.4 \pm 2.3$ & $<0.01$ \\
\hline Circumferential Basal Strain & $-22.8 \pm 4.5$ & $-18.1 \pm 5.6$ & $<0.01$ \\
\hline Circumferential Mid-ventricular Strain & $-20.5 \pm 3.6$ & & \\
\hline Circumferential Apical Strain & $-24.7 \pm 5.0$ & & \\
\hline
\end{tabular}

Mean \pm standard deviation unless as indicated

Table 2 Receiver operating characteristic curves for elevated troponin and late gadolinium enhancement

\begin{tabular}{ccccccccccc}
\hline Strain & \multicolumn{4}{c}{ Elevated Troponin } & \multicolumn{4}{c}{ Late Gadolinium Enhancement } \\
\cline { 2 - 11 } & AUC & $\begin{array}{c}\text { Strain Criterion } \\
\text { Value }\end{array}$ & $\begin{array}{c}\text { Sensitivity } \\
(\%)\end{array}$ & $\begin{array}{c}\text { Specificity } \\
(\%)\end{array}$ & $\begin{array}{c}p- \\
\text { value }\end{array}$ & $\begin{array}{c}\text { AUC } \\
\text { Value }\end{array}$ & $\begin{array}{c}\text { Strain Criteria } \\
\text { Sensitivity } \\
(\%)\end{array}$ & $\begin{array}{c}\text { Specificity } \\
(\%)\end{array}$ & $\begin{array}{c}p- \\
\text { value }\end{array}$ \\
\hline Longitudinal 4C & 0.81 & -15.83 & 83 & 84 & $<0.01$ & 0.79 & -15.5 & 72 & 86 & $<0.01$ \\
\hline Longitudinal 2C & 0.69 & -14.4 & 56 & 84 & 0.03 & 0.72 & -14.4 & 61 & 86 & 0.01 \\
\hline Global Circumferential & 0.86 & -20.8 & 83 & 74 & $<0.01$ & 0.83 & -20.5 & 83 & $<0.01$ \\
\hline Circumferential Basal & 0.77 & -24.2 & 100 & 58 & $<0.01$ & 0.76 & -24.2 & 100 & 52 & $<0.01$ \\
\hline $\begin{array}{c}\text { Circumferential Mid- } \\
\text { ventricular }\end{array}$ & 0.77 & -20.8 & 94 & 63 & $<0.01$ & 0.75 & -19.8 & 89 & 67 & $<0.01$ \\
\hline Circumferential Apical & 0.81 & -21.1 & 67 & 84 & $<0.01$ & 0.80 & -21.8 & 83 & 67 & $<0.01$ \\
\hline
\end{tabular}

\section{Authors' details}

'Pediatric Cardiology, Mount Sinai Hospital / Icahn School of Medicine, New York, NY, USA. ²Division of Pediatric Cardiology, Stony Brook Children's Hospital, Stony Brook, NY, USA. ${ }^{3}$ Department of Cardiology, Mount Sinai School of Medicine, New York, NY, USA.

Published: 3 February 2015

doi:10.1186/1532-429X-17-S1-P321

Cite this article as: Weigand et al:: Feature tracking derived longitudinal and circumferential myocardial strain abnormalities in clinical myocarditis. Journal of Cardiovascular Magnetic Resonance 2015 17(Suppl 1):P321.

\section{Submit your next manuscript to BioMed Central} and take full advantage of:

- Convenient online submission

- Thorough peer review

- No space constraints or color figure charges

- Immediate publication on acceptance

- Inclusion in PubMed, CAS, Scopus and Google Scholar

- Research which is freely available for redistribution

Submit your manuscript at www.biomedcentral.com/submit 